\title{
Características de los Intereses Vocacionales de los alumnos del 2 do y 3 er Año de la Escuela de Ingeniería en Informática y Sistemas de la Facultad de Ciencias de la Universidad Nacional Jorge Basadre Grohmann de Tacna - Año Académico 2009
}

Characteristics of Vocational Interests in students of the second and third year of the Engineering School in Computer science and Systems in the Sciences Faculty at the National University Jorge Basadre Grohmann in Tacna - Academic Year 2009

'Humberto Benito Vargas Pichón. ${ }^{2}$ Ita Daneri Huamán Guzmán

RESUMEN:

El presente Trabajo de Investigación es un estudio Exploratorio de iipo descriptivo, de corte transversal, que se efectuó durante el periodo Abril 2008 a Abril 2009 en la Escuela de Ingeniería en Informática y Sistemas (ESIS) de la Facultad de Ciencias (FACI) de la Universidad Nacional Jorge Basadre Grohmann (UNJBG) de Tacna. Mediante el objetivo general se pretendió "Identificar las características de los Intereses Vocacionales de los alumnos segundo y tercer año de la ESIS de la FACI de la UNJBG de Tacna - año académico 2009" en respuesta a la interrogante: ¿Cuáles son las características de los Intereses Vocacionales de los alumnos segundo y tercer año de la ESIS de la FACI de la UNIBG de Tacna - año académico 2009?. El diseño muestral es probabilístico sistemático y considerando el muestreo estratificado se trabajó sobre una población de 254 alumnos y con una muestra de 39 alumnos del segundo y tercer año de la ESIS de la FACI de la UNJBG de Tacna - año académico 2009. Se utilizó el Programa Excel XP 2007 para el procesamiento de datos y la elaboración de los cuadros y gráficos estadísticos, lo que nos permitió obtener la conclusión principal correspondiente.

Palabras clave: interés vocacional, carrera profesional.

\section{ABSTRACT:}

The present Work of Investigation is an Exploratory study of descriptive type, of cross section, which was done from April 2008 to April 2009 in the Engineering School in Computer science and Systems (ESIS) of the Sciences Faculty (FACI) at the National University Jorge Basadre Grohmann (UNJBG) in Tacna. By means of the general goal it was tried to "Identify the characteristics of the Vocational Interests of the students of the second and third year of the ESIS of the FACI at the UNJBG in Tacna-academic Year 2009" in answering to the question: Which are the characteristics of the Vocational Interests of the students of second and third year of the ESIS of the FACI at the UNJBG in Tacna-academic year 2009?. The sample designed is probabilistic systematic and considering the stratified sampling it was worked with a 254 students and with a 39 students sample of the second and third year of the ESIS of the FACI at the UNJBG in Tacna-Academic Year 2009. Microsofi Excel XP Program 2007 was used for the data processing and the statistical elaboration of pictures and graphs, which allowed us to obtain the corresponding main conclusion.

Keywords: vocational interests, professional career.

\footnotetext{
'Magister en Ciencias de la Computación, Magister en Educación con mención en Docencia y Gestión Educativa, Licenciado en Matemáticas Facultad de Ciencias. Universidad Nacional Jorge Basadre Grohmann

2Licenciada en Obstetricia. Ministerio de Salud - Centro de Salud de la Provincia de Tarata - Tacna
} 


\section{INTRODUCCIÓN}

Desde nuestra labor como docente de la Escuela de Ingeniería en Informática y Sistemas (ESIS) de la Facultad de Ciencias (FACI) de la Universidad Nacional Jorge Basadre Grohmann (UNJBG) vemos que alumnos de dicha Escuela no tienen claro sus intereses vocacionales en la elección de la Carrera Profesional, tal es así que tratan de seguir una especialidad que tal vez no es afin con sus potencialidades y habilidades personales; siendo esta la razón por la cual no obtienen un buen rendimiento acadénico.

La determinación de las "Características de los Intereses Vocacionales de los alumnos del segundo y tercer año de la Escuela de Ingeniería en Informática y Sistemas de la Facultad de Ciencias de la Universidad Nacional Jorge Basadre Grohmann de Tacna - Año académico 2009", permitirá dar criterios a tomar en cuenta para mejorar el servicio y la Calidad Educativa en la ESIS de la FACI de la UNJBG de Tacna.

\section{Sobre Orientación Vocacional}

La elección de una profesión y/o trabajo apunta no solo hacia una actividad u opción profesional, sino a una forma de vida, por tanto, la elección debe hacerse consciente de que con ella formamos parte de nuestra identidad, de nuestro "yo" y que a través de ella. asumimos un rol, un estatus y hasta elegimos una pareja (Aguirre Baztán, 1996).
La vocación no aparece como algo puntual y espontáneo, sino que se inicia en la infancia, va configurándose durante la adolescencia para definirse en la adultez. No obstante, estas vocaciones tempranas pueden estar enmascaradas de motivos inconscientes que no son sino compensaciones, mecanismos de defensa ante conflictos de la primera infancia; por ello es necesaria una buena orientación para realizar una elección conforme al "yo" real del sujeto (Aguirre Baztán, 1996).

Asimismo, la orientación profesional tiene como fin asesorar y ayudar al individuo a descubrir su vocación y orientarle hacia la actividad cultural o profesional en la que mejor puede realizarla, ayudando a reconocer sus propias aptitudes y asesorándole sobre cual ha de ser su preparación, no solo para realizar ese trabajo de forma efectiva, sino para poder permanecer en él. (Aguirre Baztán, 1996).

\section{METODOLOGIA}

2.1 Variable de estudio: Interés Vocacional

2.2 Tipo de estudio: El presente estudio es una investigación de tipo descriptivo transversal.

2.3 Diseño del estudio: El diseño adecuado para esta investigación es No Experimental.

Tabla 1: Intereses vocacionales de los alumnos del $2^{\circ}$ año de la ESIS-FACI-UNJBG

\begin{tabular}{l} 
Características \\
\hline Tu preocupación son los demás. Te gusta involucrarte en \\
actividades en beneficio de aquellos que necesitan ayuda: \\
enfermos, niños y ancianos abandonados. \\
Te gusta y te esfuerzas por resolver problemas numéricos. \\
En el colegio le encontrabas más satisfacción a la \\
resolución de los problemas de matemática antes que a las \\
demás materias.
\end{tabular}

Te gusta asistir a conciertos, sabes tocar un instrumento o cantar. Con frecuencia lees sobre la música y sobre la vida de los músicos.

Investigas el porqué de las cosas; descubrir las causas, los principios y reglas que explican un hecho te produce satisfacción. No importa que no obtengas ningún beneficio material de ello.

Te satisface tratar con las personas y procuras convencerlas con algún proyecto. En el colegio seguro eras el que discutía en clase o el que se encargaba de organizar y dirigir los festivales, encuentros deportivos o excursiones.
Intereses Vocacionales Total

Sacerdotes, Pedagogos, Médicos y

Cirujanos, enfermeras, Consejeros.

Auditores, Contadores Públicos,

\begin{tabular}{lll}
\hline Economistas, & & Estadísticos, \\
\hline Matemáticos & y & Funcionarios \\
\hline
\end{tabular}

Bancarios.

Compositores, Músicos, Profesores de música, Artistas de ballet.

Antropólogos,

Astrónomos, Biólogos, Ingenieros electrónicos, Ingenieros Químicos, Médicos, Odontólogos, Psicólogos, Químicos Farmacéuticos y Técnicos de Laboratorio.

Escritores, Juristas (abogados, jueces, consejeros jurídicos) Jefes de ventas, Locutores de radio y $\mathrm{TV}$.

Fuente: Elaboración propia 
Tabla 2. Intereses vocacionales de los alumnos del 3 er año de la ESIS-FACI-UNJBG

\begin{tabular}{l} 
Características \\
\hline Investigas el porqué de las cosas; descubrir las causas, los \\
principios y reglas que explican un hecho te produce \\
satisfacción. No importa que no obtengas ningún \\
beneficio material de ello.
\end{tabular}

Tu preocupación son los demás. Te gusta involucrarte en actividades en beneficio de aquellos que necesitan ayuda: enfermos, niños y ancianos abandonados

Te gusta asistir a conciertos, sabes tocar un instrumento o cantar. Con frecuencia lees sobre la música y sobre la vida de los músicos

Te gusta y te esfuerzas por resolver problemas numéricos. En el colegio le encontrabas más satisfacción a la resolución de los problemas de matemática antes que a las demás materias. + INTERES MUSICAL: Te gusta asistir a conciertos, sabes tocar un instrumento o cantar. Con frecuencia les sobre la música y sobre la vida de los músicos.

Te gusta y te esfuerzas por resolver problemas numéricos. En el colegio le encontrabas mas satisfacción a la resolución de los problemas de matemática antes que a las demás materias

Te gusta llevar un registro metódico de gastos, tienes tus cuadernos ordenados y tus libros bien forrados. Te gusta guardar documentos y clasificarlos

Te satisface tratar con las personas y procuras convencerlas con algún proyecto. En el colegio seguro que eras el que discutía en clase o se encargaba de organizar y dirigir los festivales, encuentros deportivos o excursiones.

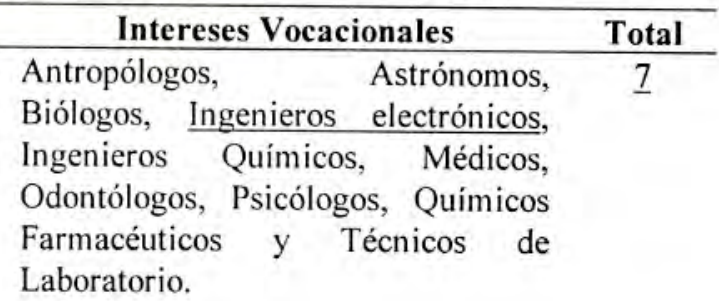

Sacerdotes, Pedagogos, Médicos y

Cirujanos, enfermeras, Consejeros.

Compositores, Músicos, Profesores

de música, Artistas de ballet.

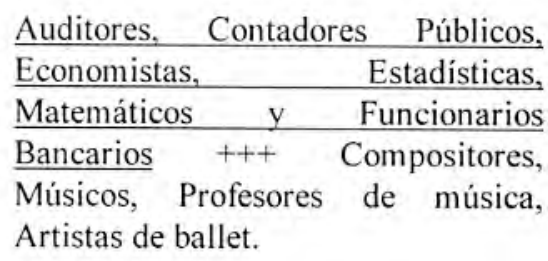

\begin{tabular}{lr}
\hline Economistas, & Estadísticas, \\
\hline Matemáticos y & Funcionarios \\
\hline Bancarios +++ & Compositores, \\
$\begin{array}{l}\text { Músicos, Profesores de música, } \\
\text { Artistas de ballet. }\end{array}$
\end{tabular}

Auditores, Contadores Públicos,

\begin{tabular}{lll}
\hline Economistas, & Estadisticos, \\
\hline Matemáticos y y & Funcionarios \\
Bancarios. & &
\end{tabular}

Archivistas, Contadores, Secretarias y Bibliotecarios.

Escritores, Juristas (Abogados, Jueces, Consejeros juridicos), Agentes de publicidad, Jefe de ventas, Locutores de radio y $\mathrm{TV}$.

TOTAL

Fuente: Elaboración propia

3.4 Téenica e instrumento de recolección de datos: se utilizó la técnica de la encuesta y los datos fueron obtenidos mediante la aplicación de un cuestionario que nos permitió conocer los Intereses Vocacionales de los alumnos confortantes de la muestra.

\section{RESULTADOS}

VerTabla 1 y Tabla 2.

\section{CONCLUSIONES}

1. Solo el $49 \%$ de los alumnos encuestados tienen bien definido sus intereses vocacionales de acuerdo a la elección de su carrera profesional.

2. En consecuencia, existe un $51 \%$ de los alumnos encuestados que NO tienen bien definido sus intereses vocacionales en concordancia a la elección de su carrera profesional

\section{RECOMENDACIONES}

Que investigaciones futuras relacionadas con la presente investigación, puedan ser ampliadas y de tipo Experimental, para mejorar las condiciones de desarrollo personal de los estudiantes de la Escuela Académico Profesional de Ingeniería en Informática y Sistemas de la Universidad Nacional Jorge Basadre Grohmann de Tacna.

Creación de un área de Orientación Vocacional en donde se planifique y se implemente la evaluación sobre orientación vocacional para potenciar las capacidades de los estudiantes de la Escuela Académico Profesional de Ingeniería en Informática y Sistemas de la Universidad Nacional Jorge Basadre Grohmann de Tacna. 
Explicar a los padres de familia sobre los intereses vocacionales de sus hijos, con el objeto que les brinden el apoyo necesario a fin de obtener mejores resultados de ellos.

Los padres de familia deben colaborar y participar en el proceso de orientación de sus hijos, siendo debidamente informados de la realidad educativa y laboral existente para aconsejar y apoyar a sus hijos, siempre y cuando no haya interferencia en la libre elección de los mismos.

\section{REFERENCIAS BIBLIOGRÁFICAS}

\section{Fuentes Bibliográficas}

Herzberg, Frederick. (1971). "Administradores o entrenadores de animales". Revista de administración.

Maslow, Abraham. (1954). "Motivación y personalidad". Harper \& Row.

McGregor, Douglas. (1960). "El lado humano de la empresa". McGraw-hill.

Robbins, Stephen. (1999). "Comportamiento Organizacional". Prentice Hall. $8^{\circ}$ Edición.

\section{Fuentes Hemerográficas}

Chacon, O. (2003). Diseño, aplicación y evaluación de una propuesta de orientación vocacional para la Educación Media, Diversificada y Profesional venezolana. Trabajo de Grado, Programa de doctorado: innovación y sistema educativo, Universitat Rovira i Virgili, San Cristóbal-Venezuela.
Zavala, G. (2001). El clima familiar, su relación con los intereses vocacionales y los tipos caracterológicos de loa alumnos del 5to. Año de secundaria de los colegios nacionales del distrito del Rimac. Trabajo de Licenciatura, Licenciatura en Psicología, Universidad Nacional Mayor de San Marcos, Lima-Perú.

\section{Fuentes Electrónicas}

Cabrera, P. (s/f). "Orientación Vocacional y Profesional". Recuperado de

http://www.rie.cl/orientacion_vocacional.php [2008: 13 de Abril]

Aceros, J., Angarita S. y Campos O. Depresión y Rendimiento académico, recuperado de, http://www.psicopedagogia.com/articulos/?articulo=31 1 [2007: 08 de Enero].

\section{Correspondencia:}

Humberto Vargas Pichon

Ciudad Universitaria Fundo "Los Granados"

Av. Miraflores s/n. Tacna. Perú

humberto.vargas@hotmail.com

humbero.vargas@peru.com

humberto.vargas@unjbg.edu.pe

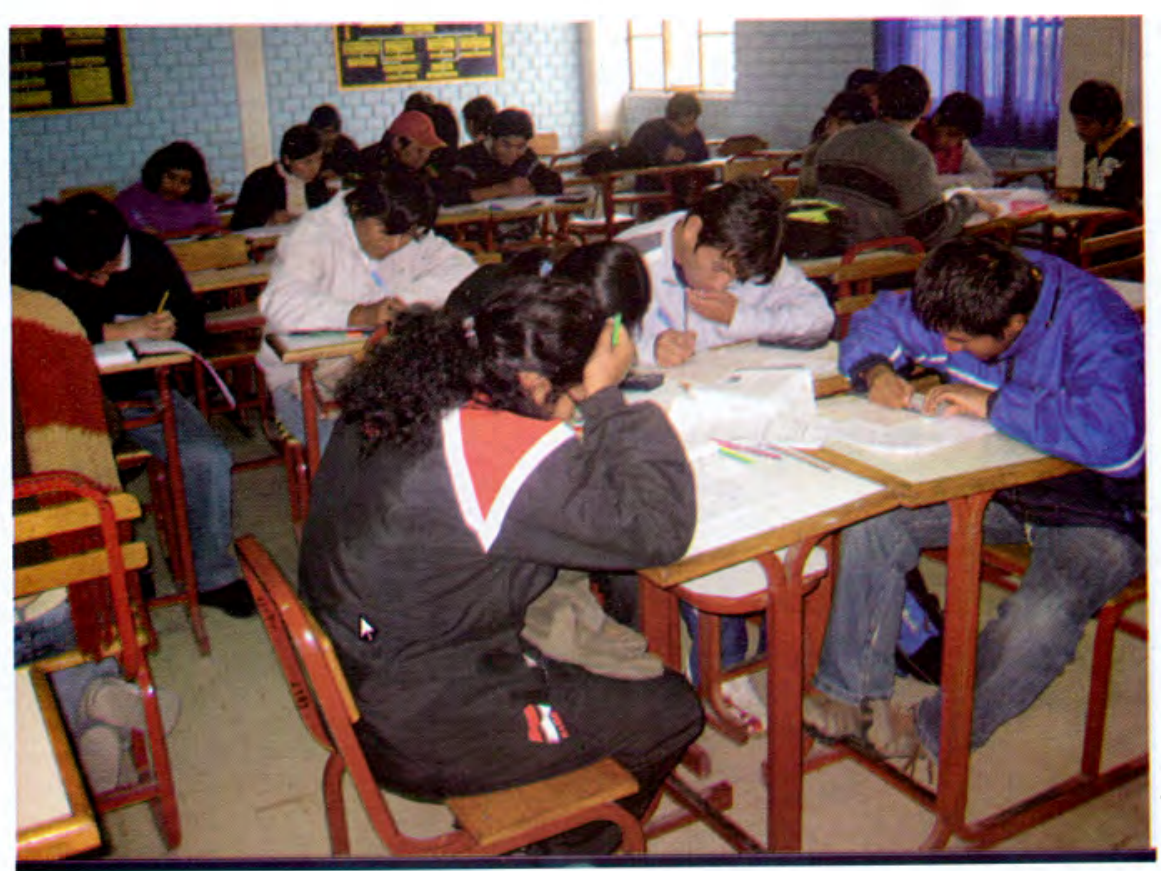

Estudiantes de la Escuela de Ingeniería en Informática y Sistemas de la Facultad de Ciencias de la UNJBG en Encuesta de Interés Vocacional, Tacna 2009 